\title{
Lderazgo transformacional de los directivos y su relación con el compromiso organizacional de los docentes en los Colegios Públicos de la Provincia de San Martin, en el año 2017.
}

Transformational leadership of managers and their relationship with the organizational commitment of teachers in the Public Schools of the Province of San Martin, in 2017

\author{
Elmer Cruzado Vásquez \\ Unidad de Posgrado de Ciencia Empresariales, Universidad Peruana Unión
}

INFORMACIÓN DEL ARTÍCULO

Historia del artículo

Recibido:

Aceptado:

\section{Palabras clave:}

Liderazgo

liderazgo,

organizacional; organización.

\section{Resumen}

El objetivo de esta investigación fue determinar la relación entre el liderazgo transformacional de los directivos y el compromiso organizacional de los docentes en los colegios públicos de la provincia de San Martin, en el año 2017. Dicha investigación es de tipo descriptiva - correlacional con un diseño no experimental transversal, la muestra estuvo conformada por los docentes de los colegios públicos de la provincia de San Martin haciendo un total de 363 encuestados. Los instrumentos aplicados para este estudio fueron; para la escala de liderazgo transformacional el instrumento empleado por Minaya (2014), con una escala de tipo Likert; y para medir el compromiso organizacional, se utilizó el cuestionario de Meyer y Allen aplicado por Zegarra (2014), ambos instrumentos cuentan con la validación a juicio de experto. La confiabilidad del instrumento tanto para el Liderazgo Transformacional como para el Compromiso Organizacional fue medida gracias al por el alfa de Cronbach con un valor de 0.862, y un valor de 0.610, respectivamente, en ambos casos indica fiabilidad aceptable. Los resultados concluyen que existe relación significativa entre el Liderazgo Transformacional de los Directivos y el Compromiso Organizacional de los Docentes en los Colegios Públicos de la Provincia de San Martin, ya que se obtuvo un coeficiente Rho de Spearman igual a 0,199 y un $p$ valor igual a 0,001.

\section{Abstract}

The objective of this research was to determine the relationship of, transformational leadership of managers and their relationship with the organizational commitment of teachers in public schools in the province of San Martin, in the year 2017. This research is descriptive - correlational with a non- experimental transversal design, the sample consisted of teachers from public schools in the province of San Martin, making a total of 363 respondents. The instruments applied for this study were; for the transformational leadership scale, the instrument used by Minaya (2014) was used. Likert scale; and to measure the organizational commitment, the questionnaire of Meyer and Allen applied by Zegarra (2014) was used, both instruments had the approval of experts. The reliability of the Transformational Leadership instrument was through Cronbach's alpha with a value of 0.862 , and for the organizational commitment instrument it was also through Cronbach's alpha which had a value of 0.610 , which in both cases indicates acceptable reliability. The results conclude that there is a significant relationship between the Transformational Leadership of the Directors and the Organizational Commitment of the Teachers in the Public Schools of the Province of San Martin, since a Rho coefficient of Spearman equal to 0.199 and a $p$ value equal to 0.001 was obtained.
Keywords:

leadership,

commitment; organization. leadership; organizational 


\section{Introducción}

De acuerdo con cifras de la Comisión Económica para América Latina y el Caribe (CEPAL) y la Superintendencia Nacional de Aduanas y Administración Tributaria (SUNAT), Moran y Pecho (2016) el Perú es uno de los países con un alto grado de evasión tributaria, alcanzando un 34,7\% (Quintanilla, 2014).

Actualmente, en el mundo de los negocios el activo más importante para las organizaciones es el talento humano, esto hace que la alta dirección le dé mucho valor a su estudio y a la manera como debe ser gestionado el talento que poseen los trabajadores. A razón de ello se vive un panorama en el cual es alto el nivel de exigencia de los procesos en pro del cumplimiento de los estándares de calidad de las compañías, en las cuales el liderazgo que posean los administradores, juega un papel importante y trascendental, esto hace que los estudios en este campo tomen cada vez más fuerza. Según investigaciones, han pasado muchos años desde que Burns en 1978 citara de manera preliminar en su curso de trabajo, las definiciones de liderazgo transaccional y transformacional (Bass et al., 2006).

De este modo, es una realidad que la gestión de la organización descansa en el liderazgo que ejercen sus directivos, esto indica que es trascendental en la conducción de la misma, que sin duda se reflejará en el desempeño de sus trabajadores. Sin embargo, el éxito de una organización no solo radica en el buen liderazgo de sus administradores, sino también en el compromiso que tienen los trabajadores con su centro de labores.

Es así como la Gestión del Talento Humano en la actualidad enfrenta a un gran reto, reto que implica la creación de méto- dos o herramientas útiles los cuales puedan comprometer al individuo con las metas que persigue la institución, buscando la lealtad por parte de sus trabajadores; el departamento de Gestión del Talento Humano (GTH), utiliza el mecanismo de compromiso organizacional (Bayona, Legaz, y Madorrán, 1999). Es en este contexto Minaya (2014), afirma que el sector educación no es ajeno a las exigencias que se plantean anteriormente ya que en los últimos años la educación a nivel mundial ha sufrido cambios que han transformado la forma de dirigirla. Es por ello que este artículo se centra en el estudio del liderazgo transformacional y el compromiso organizacional, puesto que son variables sumamente relevantes para el éxito de cualquier organización.

\section{Referencias teóricas}

\section{Liderazgo.}

Chiavenato (2006), refiere que el liderazgo es un proceso de influenciar sobre un individuo o un conjunto de individuos que se esfuerzan por lograr sus metas en determinada situación. Por su parte Castro, Clemenza y Araujo (2012), mencionan que el Liderazgo es un proceso natural de influencia que ocurre entre una persona que lidera y otra que lo sigue. Esto indica que es una relación recíproca y de influencia que se da entre los líderes y liderados en la cual las ambas partes pretenden obtener a cambios reales.

\section{Importancia del liderazgo.}

El liderazgo es un elemento esencial en estos días. Los líderes nacen o se construyen a lo largo de su vida. En toda organización se ha encontrado a personas que inspiran a los demás a perseguir sus sueños hasta lograrlos y que además estos sueños que posee deben estar alineados 
a las metas de la empresa (Grijalva, 2009). Para Noriega (2008), una empresa puede tener el control adecuado, una planeación adecuada, etc. y no permanecer en el mercado a la falta de un líder, incluso dicha empresa puede carecer de planeación y control, pero, si cuenta con el apoyo de su líder tendría el éxito asegurado. Es por ello que es de vital importancia un líder dentro de las organizaciones.

\section{Liderazgo transformacional.}

Este tipo de liderazgo se ha catalogado como un tema de mucho interés que es abordado en estudios y proyectos de diversos sectores. Aunque su introducción en el sector educación es completamente nueva, algunos estudios indican que es el más adecuado para el sistema educativo, y debido a ello esta teoría ofrece mucho al campo empresarial y educativo. Este aporte es gracias a que el liderazgo transformador trabaja con las personas y su mayor interés está en el bienestar de su gente. Estos hombres inspiran y transforman a sus trabajadores sobrepasando sus expectativas conduciéndolos a alcanzar de sus objetivos (Minaya, 2014).

Dimensiones de liderazgo transformacional.

El liderazgo carismático está conformado por componentes o también denominado como dimensiones que su estudio es vital, estas dimensiones son (Minaya, 2014).

- La consideración individual.

- La estimulación intelectual.

- La motivación inspiradora y

- La tolerancia psicológica.

Compromiso organizacional.

El compromiso que posee el individuo con la organización donde labora, es abordado por diversos autores, logrando así tener enfoques que ayudan a aclarar el panorama. El compromiso organizacional ha sido considerado como un término que se ha utilizado con sentidos muy diversos (Robles y De la Garza, 2009). Becker (como se citó en Díaz, Palos, y Paz, 2006), ha definido al compromiso como el vínculo que establece el individuo con su organización, fruto de pequeñas recompensas adquiridas a lo largo de los años. Es así como el compromiso organizacional se ha convertido en una poderosa herramienta de gestión de los Recursos Humanos en la actualidad.

Dimensiones del compromiso organizacional.

Según el modelo de Meyer y Allen (Mehech, Cordero, y Gómez, 2016) el compromiso organizacional tiene tres componentes:

- Compromiso afectivo

- Compromiso continuo y

- El compromiso normativo.

\section{Materiales y métodos}

La presente investigación se encuentra bajo el enfoque cuantitativo, dentro del diseño descriptivo, correlacional - causal debido a que se analizará la relación de múltiples variables (independientes) frente al compromiso organizacional, donde según (Hernández, Fernández, y Baptista (2014) radica en encontrar la relación que pueda existir entre dos variables. En este sentido, la investigación corrobora si el liderazgo transformacional de los directivos afecta de manera positiva y directa en el compromiso que sus docentes tienen con la organización. Este estudio es abordado a través de un diseño no 
experimental, de corte transversal. No experimental, debido a que no se manipuló ni a las variables ni a los sujetos que conformaran la muestra; se observó y evaluaron los datos tal como se manifestaron, $y$ transversal puesto que la recolección de datos se hizo en una sola ocasión, por lo que los participantes del estudio no tuvieron que responder, más de una vez, a las preguntas establecidas en el instrumento. La investigación presenta el estudio y análisis minucioso de dos variables 1) Liderazgo transformacional de los directivos y 2) Compromiso Organizacional de los docentes, en el cual se observaron y midieron las variables con la finalidad de determinar si existe relación estadísticamente significativa entre ambas variables o no.

\section{Participantes.}

Población y muestra de acuerdo con Carrasco (citado en Salvador y Sánchez, 2018), quienes mencionan que es el conjunto de todos los elementos (unidades de análisis) que conforman el espacio donde se ejecutará el trabajo de investigación. En este sentido, la población comprende a los participantes en estudio, la cual está conformada por todos los docentes que forman parte de los colegios públicos en la Provincia de San Martin. En los 14 distritos existen 49 colegios públicos, en éstos laboran un total de 829 docentes los cuales conforman la población del estudio. De esta población salió la muestra, que para efectos de la investigación fueron 263 docentes.

Ahora bien, para el estudio se consideró a los docentes nombrados y contratados de los Colegios Públicos, de los 14 distritos de la Provincia de San Martin; la muestra estuvo conformada por 263 maestros y fue elegida aplicando el método no probabilístico por conveniencia, que consiste en la elección arbitraria de las unidades muéstrales que el investigador considere adecuados para proporcionar la información además de la disposición para responder satisfactoriamente a las preguntas del instrumento.

\section{Instrumentos.}

Las técnicas a utilizar en este estudio fueron de dos tipos: Técnicas de gabinete, la cual consiste en realizar una revisión bibliográfica para el desarrollo del marco teórico y para el estudio de campo, se emplearon dos instrumentos validados y utilizados en otras investigaciones por expertos, que conforman las dos variables de estudio:

- Instrumento sobre liderazgo transformacional.

- Instrumento sobre compromiso organizacional.

\section{Análisis de datos.}

La codificación y almacenamiento de los datos para su posterior análisis se realizaron utilizando hojas de cálculo en Excel; y para hacer un análisis eficiente de los datos se utilizó el SPSS versión 22. Una vez los datos sometido a un análisis estadístico para determinar la distribución de frecuencias de cada una de las variables utilizadas en este estudio. Para contrastar las hipótesis y el programa de SPSS se aplicó la prueba estadística de correlación de Pearson. Con respecto a la validación del instrumento a emplear se está tomando dos instrumentos que ya están validados y se está adaptando a la realidad y finalidad del estudio. Adicional a ello se trabajó también con juicio de expertos, que sin duda contribuyeron a que el instrumento adaptado tuviese un mayor grado de confiabilidad. 
El procedimiento estadístico para la relación entre Liderazgo Transformacional y Compromiso Organizacional fue Rho Spearman, debido a que la escala de Liderazgo Transformacional es de $05 \mathrm{y}$ compromiso organizacional son de $06 \mathrm{y}$ que además son medidos con una escala tipo Likert.

\section{Resultados y Discusión}

Según el análisis descriptivo se ha considerado las valoraciones de la variable $\mathrm{Li}$ derazgo transformacional de los directivos y Compromiso organizacional de los do- centes cuyos resultados obtenidos se presentan a continuación de manera clara y precisa.

En la Tabla 1. Se encuentran los resultados en los cuales se refleja que el $4 \%$ de los docentes encuestados perciben que los directivos practican a veces el Liderazgo transformacional en la Institución Educativa donde laboran, el $91 \%$ casi siempre lo practica y el $5 \%$ lo practica siempre, esta percepción es favorable para los colegios públicos que conforman la UGEL de la Provincia de San Martin.

Tabla 1

Análisis descriptivo de Liderazgo Transformacional

\begin{tabular}{lcc}
\hline Categoría & Frecuencia & Porcentaje \\
\hline A veces & 11 & 4 \\
Casi siempre & 240 & 91 \\
Siempre & 12 & 5 \\
Total & 263 & 100 \\
\hline
\end{tabular}

Fuente: elaboración propia

En la Tabla 2. Se afirma que el $1 \%$ de los docentes encuestados están en desacuerdo con el Compromiso organizacional, en la Institución Educativa donde laboran, el $68 \%$ están de acuerdo, el 30\% están muy de acuerdo y el $1 \%$ están definitivamente de acuerdo. Estos resultados demuestran que si existe compromiso de parte de los docentes con su institución o lugar de trabajo.

Tabla 2

Análisis descriptivo de Compromiso Organizacional

\begin{tabular}{lcc}
\hline \multicolumn{1}{c}{ Categoría } & Frecuencia & Porcentaje \\
\hline En desacuerdo & 3 & 1 \\
De acuerdo & 178 & 68 \\
Muy de acuerdo & 79 & 30 \\
Definitivamente de acuerdo & 3 & 1 \\
Total & 263 & 100 \\
\hline
\end{tabular}

Fuente: elaboración propia

En la Tabla 3. Se presenta la relación entre el Liderazgo Transformacional de los Directivos y el Compromiso Organizacional de los Docentes en los Colegios Públicos de la Provincia de San Martin, don- de se obtuvo un coeficiente Rho de Spearman igual a 0,199 (correlación positiva baja) y un $p$ valor igual a 0,001 (p-valor < 0.05), por lo tanto, se acepta la hipótesis alterna, es decir existe relación estadísti- 
camente significativa entre el Liderazgo Transformacional de los Directivos y el Compromiso Organizacional de los Docentes en los Colegios Públicos de la Provincia de San Martin 2017. Lo que indica que si se incrementa el nivel de liderazgo transformacional en los directivos, se logrará ligeramente un mejor Compromiso Organizacional de los Docentes.

Tabla 3

Análisis de correlación entre el Liderazgo Transformacional de los Directivos y el Compromiso Organizacional de los Docentes en los Colegios Públicos de la Provincia de San Martin, en el año 2017.

\begin{tabular}{llll} 
Liderazgo & & & \\
\cline { 2 - 4 } Transformacional & Rho Spearman & p-valor & $\mathrm{N}$ \\
\cline { 2 - 4 } & 0,199 & 0,001 & 263
\end{tabular}

Fuente: elaboración propia

En la Tabla 4. Se presenta la relación entre la Atención Individual de los Directivos y el Compromiso Organizacional de los Docentes en los Colegios Públicos de la Provincia de San Martin. Se obtuvo un coeficiente Rho de Spearman igual a 0,127 (correlación positiva baja) y un $\mathrm{p}$ valor igual a 0,039 ( $p$-valor $<0.05$ ), por lo tanto, se acepta la hipótesis alterna, es decir, existe relación estadísticamente significativa entre la Atención Individual de los Directivos y el Compromiso Organizacional de los Docentes en los Colegios Públicos de la Provincia de San Martin, en el año 2017. Es decir, al mejorar la atención individual de los directivos, se aumenta ligeramente el Compromiso Organizacional de los Docentes.

Tabla 4

Análisis de correlación entre la Atención Individual de los Directivos y el Compromiso Organizacional de los Docentes en los Colegios Públicos de la Provincia de San Martin, en el año 2017.

Compromiso Organizacional

\begin{tabular}{|c|c|c|c|}
\hline \multirow{3}{*}{ Atención Individual } & & & \\
\hline & Rho Spearman & p-valor & $N$ \\
\hline & 0,127 & 0,039 & 263 \\
\hline
\end{tabular}

Fuente: elaboración propia.

En la Tabla 5. Se presenta la relación entre la Estimulación Intelectual de los Directivos y el Compromiso Organizacional de los Docentes en los Colegios Públicos de la Provincia de San Martin, en el año 2017. Se obtuvo el coeficiente Rho de Spearman de 0,138 (correlación positiva baja) y un $p$ valor igual a 0,025 ( $p$-valor < 0.05 ), por lo tanto, se acepta la hipótesis alterna, es decir, existe relación significativa entre la Estimulación Intelectual de los Directivos y el Compromiso Organizacional de los Docentes en los Colegios Públicos de la Provincia de San Martin, en el año 2017. Es decir, al aumentar la estimulación Intelectual de los Directivos, se mejorará ligeramente el Compromiso Organizacional de los Docentes. 
Tabla 5

Análisis de correlación entre la Estimulación Intelectual de los Directivos y el Compromiso Organizacional de los Docentes en los Colegios Públicos de la Provincia de San Martin, en el año 2017.

Estimulación

Compromiso Organizacional

Intelectual

\begin{tabular}{ccc}
\hline Rho Spearman & p-valor & N \\
\hline 0,138 & 0,025 & 263
\end{tabular}

Fuente: elaboración propia

En la Tabla 6. Se presenta la relación entre la Motivación Inspiradora de los Directivos y el Compromiso Organizacional de los Docentes en los Colegios Públicos de la Provincia de San Martin. Se obtuvo el coeficiente Rho de Spearman de 0,141 (correlación positiva baja) y un $p$ valor igual a 0,022 ( $p$-valor < 0.05), por lo tanto, se acepta la hipótesis alterna, es decir, existe relación significativa entre la Moti- vación Inspiradora de los Directivos y el Compromiso Organizacional de los Docentes en los Colegios Públicos de la Provincia de San Martin, en el año 2017. Es decir, al aumentar el nivel de Motivación Inspiradora de los Directivos, el nivel de Compromiso Organizacional de los Docentes en los Colegios, se mejorará ligeramente.

Tabla 6

Análisis de correlación entre la Motivación Inspiradora de los Directivos y el Compromiso Organizacional de los Docentes en los Coleqios Públicos de la Provincia de San Martin, en el año 2017.

\begin{tabular}{cccc}
\multirow{2}{*}{$\begin{array}{c}\text { Motivación } \\
\text { Inspiradora }\end{array}$} & \multicolumn{3}{c}{ Compromiso Organizacional } \\
\cline { 2 - 4 } & $\begin{array}{c}\text { Rho } \\
\text { Spearman }\end{array}$ & p-valor & $\mathrm{N}$ \\
\cline { 2 - 4 } & 0,141 & 0,022 & 263 \\
\hline
\end{tabular}

Fuente: elaboración propia

En la Tabla 7. Se presenta la relación entre la Tolerancia Psicológica de los Directivos y el Compromiso Organizacional de los Docentes en los Colegios Públicos de la Provincia de San Martin, en el año 2017. Se obtuvo el coeficiente Rho de Spearman de 0,175 (correlación positiva baja) y un $p$ valor igual a 0,004 ( $p$-valor $<0.05$ ), por lo tanto, se acepta la hipótesis alterna, es decir, existe relación significativa entre la Tolerancia Psicológica de los Directivos y el Compromiso Organizacional de los Docentes en los Colegios Públicos de la Provincia de San Martin, en el año 2017. Al aumentar el nivel de Tolerancia Psicológica de los Directivos, el Compromiso Organizacional de los Docentes, mejorará levemente. 
Tabla 7

Análisis de correlación entre la Tolerancia Psicológica de los Directivos y el Compromiso Organizacional de los Docentes en los Colegios Públicos de la Provincia de San Martin, en el año 2017.

Tolerancia

Compromiso Organizacional

Psicológica

\begin{tabular}{ccc}
\hline Rho Spearman & p-valor & $\mathrm{N}$ \\
\hline 0,175 & 0,004 & 263
\end{tabular}

Fuente: elaboración propia

En suma, el liderazgo transformacional de los directivos es un indicador esencial en el grado de compromiso organizacional que posee el docente con su centro de labor; por consiguiente en los resultados, en respuesta a las hipótesis planteadas se observa que sí existe relación positiva entre ambas variables. Por su parte el liderazgo transformacional tiene la finalidad de generar cambios en la cultura organizativa, es el agente que transforma la cultura de la empresa (Bernal, 2000).

En este sentido, se aceptó la hipótesis alterna y se rechazó la hipótesis nula, lo cual indicaría que al aumentar el grado de liderazgo transformacional el compromiso organizacional de los docentes aumenta. A si también Sun y Liethwood (2012), refieren que el propósito del liderazgo es que sus trabajadores se sientan motivados con su labor y en que sus metas sean colectivas, pero que además sientan motivación hacia el logro y la autorrealización. Con los resultados de esta investigación, y gracias al respaldado de distintos autores que dan soporte teórico a este estudio, se pudieron proponer algunas recomendaciones de mejora en el sector educativo de los colegios públicos que conforman la UGEL San Martin. Por otro lado, de acuerdo con los resultados también se observa que el compromiso organizacional de los docentes es mediamente favorable con la institución, que hace que sus habilidades y conocimientos, sea un poco difícil de alinearlos con los objetivos que persiguen los directivos para el colegio. Sin embargo, sería favorable que se haga hincapié en el aporte que tienen muchos especialistas para la gestión de la organización. Esto concuerda con lo que dice Bennetts (2007), que se debe partir del tipo de liderazgo transformacional en la Institución, un director líder con mentalidad innovadora con toma de decisiones en colectivo con una visión al futuro concreto.

De la misma forma, se encuentra la conclusión de Almirón, Tikhomirova, Trejo, y García (2015), quienes mencionan que el impacto ejercido por el liderazgo a nivel organizacional es fundamental, ya que es considerado un pilar para triunfo organizacional. Por lo tanto, los resultados del estudio plantean que existe relación positiva entre ambas variables.

\section{Conclusiones}

De acuerdo con los hallazgos en el estudio de la relación entre el Liderazgo Transformacional y el Compromiso Organizacional de los docentes, se han elaborado las siguientes conclusiones:

Se determinó la relación existente entre el Liderazgo Transformacional de los 
Directivos y el Compromiso Organizacional de los Docentes en los Colegios Públicos de la Provincia de San Martin, en el año 2017. La medición de estas variables ha permitido determinar que sí existe relación estadísticamente significativa entre el Liderazgo Transformacional de los Directivos y el Compromiso Organizacional de los Docentes en los Colegios Públicos de la Provincia de San Martin. Por lo que a medida que se incremente el nivel de liderazgo transformacional en los directivos, se logrará un mejor Compromiso Organizacional de los Docentes.

Asimismo Al medir la variable de Liderazgo Transformacional de los directivos en los colegios Públicos de la Provincia de San Martin, se ha evidenciado que el $91 \%$ de los encuestados perciben que sus directivos casi siempre practican el Liderazgo Transformacional, mientras que un $5 \%$ indican que los directivos siempre practican un liderazgo Transformacional. Del mismo modo, se ha encontrado que el $68 \%$ de los encuestados están de acuerdo con el Compromiso Organizacional en la institución donde laboran, así mismo el $30 \%$ están muy de acuerdo con el compromiso organizacional, lo cual indica que el $98 \%$ los docentes encuestados tienen compromiso con la I.E. donde laboran.

Además, al determinar la relación que existe entre la Consideración Individual de los Directivos y el Compromiso Organizacional de los Docentes en los Colegios Públicos de la Provincia de San Martin, en el año 2017, se demostró que existe relación estadísticamente significativa entre la Consideración Individual de los Directivos y el Compromiso Organizacional de los Docentes. Es decir, al mejorar la atención individual de los directivos, se aumenta ligeramente el Compromiso Organizacional de los Docentes.
Notablemente, se determinó la relación que existe entre la Estimulación Intelectual de los Directivos y el Compromiso Organizacional de los Docentes en los Colegios Públicos de la Provincia de San Martin, en el año 2017, llegando a concluir que sí existe relación significativa entre la Estimulación Intelectual de los Directivos y el Compromiso Organizacional de los Docentes, ya que se obtuvo el coeficiente Rho de Spearman de 0,138 (correlación positiva baja) y un $p$ valor igual a 0,025 ( $p$ valor $<0.05$ ) con lo que se podría afirmar que a mayor Estimulación intelectual, mayor será el Compromiso Organizacional de los docentes.

De igual forma, en la dimensión Motivación inspiración de la variable Liderazgo Transformacional Se relaciona de manera significativa con el Compromiso Organizacional de los Docentes en los Colegios Públicos de la Provincia de San Martin, debido a que se obtuvo el coeficiente Rho de Spearman de 0,141 (correlación positiva baja) y un $p$ valor igual a 0,022 ( $p$-valor < 0.05 ), por lo tanto, existe relación significativa entre la Motivación Inspiradora de los Directivos y el Compromiso Organizacional de los Docentes, por lo que, que aumente el nivel de Motivación Inspiración de los Directivos, el nivel de Compromiso Organizacional de los Docentes en los Colegios incrementaría.

Finalmente, se concluye que sí existe relación estadísticamente significativa entre la Tolerancia Psicológica de los Directivos y el Compromiso Organizacional de los Docentes en los Colegios Públicos de la Provincia de San Martin, en el año 2017. Todo esto debido a las indagaciones empíricas donde se obtuvo el coeficiente Rho de Spearman de 0,175 (correlación positiva baja) y un $p$ valor igual a 0,004 ( $p$-valor < 0.05), esto quiere decir 
que, al aumentar el nivel de Tolerancia Psicológica de los Directivos, el Compromiso Organizacional de los Docentes, mejorará levemente.

\section{Recomendaciones}

Después de tener los resultados y las conclusiones a las que se ha llegado en el estudio se han planteado las siguientes recomendaciones o sugerencias.

Primera. Mejorar el Compromiso Organizacional de los docentes perfeccionando el Liderazgo Transformacional de los directivos mediante una serie de capacitaciones que muestren los beneficios que se obtendría para la institución educativa el ejercer este tipo de liderazgo, además con el objetivo de mejorar el desempeño docente y que este se identifique con la institución donde labora.

Segunda. Mejorar la Atención Individual mediante el mejoramiento del Liderazgo Transformacional de los directivos, implementando un plan de monitoreo hacia los docentes logrando de esa manera conocer sus necesidades, sugerencias y temores, logrando así conocerlos más de cerca para luego satisfacer sus necesidades tanto personales como laborales y con ello obtener un mayor compromiso con la institución.
Tercera. Mejorar la Motivación Inspiración a través del perfeccionamiento del Liderazgo Transformacional de los directivos, exponiendo a los docentes cada vez que sea posible el plan estratégico que persigue la institución (misión, visión, objetivos, FODA, MOF), permitiendo que éstos se comprometan y sean parte de las metas organizacionales, logrando con ello que los maestros se sientan importantes e identificados con la institución educativa donde laboran.

Cuarta. Realizar mejoras en el Liderazgo Transformacional de los directivos a fin de mejorar la Tolerancia Psicológica hacia los docentes, esto será posible en la medida en la que los directivos interioricen y reconozcan la importancia y beneficios que trae el ser tolerantes con los errores de los demás. Por otro lado, se sugiere que los directores muestren empatía y aprecio con sus trabajadores ya que de esto dependerá en gran medida la confianza que muestre el maestro para hacer su trabajo.

Quinta. Se sugiere realizar nuevas investigaciones similares en instituciones educativas del sector privado y otros sectores no educativos en las que se relacione el Liderazgo Transformacional de los directivos y el Compromiso Organizacional. 


\section{Referencias}

Almirón, V., Tikhomirova, A., Trejo, A., \& García, J. (2015). Liderazgo transaccional vs Liderazgo transformacional. ReiDoCrea, 4, 24-27. Retrieved from http://digibug.ugr.es/bitstream/handle/10 481/34629/AlmironArevalo_V4_Art4.pdf ?sequence $=6$ \&isAllowed $=y$

Bass, B. M., Studies, L., Alejandro, I., Martínez, M., María, L., Ortiz, F., Salle U. La. (2006). desarrollo en Liderazgo Transformacional. Leadership, 7(1), 2542. Retrieved from http://www.redalyc.org/pdf/342/3420270 2.pdf

Bayona, C., Legaz, S., \& Madorrán, C. (1999). Compromiso organizacional: implicaciones para la gestión estratégica de los recursos humanos, (January 2000). Retrieved from https://www.researchgate.net/profile/Cri stina_Bayona/publication/266454597_C ompromiso_Or ganizacional_Implicaciones_para_la_ge stin_estratgica_de_los_Recursos_Huma nos/links/54b65 0b30cf2bd04be31fb00.pdf

Bennetts, S. (2007). El liderazgo transformacional y la evaluación de programas académicos universitarios en México. Director, 163-189. Retrieved from

http://www.redalyc.org/pdf/3421/342130 829008.pdf

Bernal, J. L. A. (2000). Liderar el cambio: El liderazgo transformacional. Anuario de Pedagogía, 198-199. Retrieved from http://didac.unizar.es/jlbernal/articulos_p ropios/pdf/02_lidtrans.PDF

Castro, E., Clemenza, C., \& Araujo, R. (2012) Líderes y seguidores : una relación dual. Multiciencias, 12, 251-255. https://doi.org/90431109041
Chiavenato, I. (2006). Introducción a la Teoría General de la Administración. (N. islas López, Ed.), Mc Graw Hill Interamericana (Séptima ed). Delegación Cuajimalpa, México, D. F. Retrieved from https://naghelsy.files.wordpress.com $/ 20$ 16/02/introduccic3b3n-a-la-teorc3adageneral-de- la-administracic3b3n-7maedicic $3 b 3 n$-idalberto-chiavenato.pdf

Díaz, N., Palos, P., \& Paz, F. (2006). Compromiso organizacional en una muestra de trabajadores mexicanos. Articulo, 22, Págs. 25-43. ISSN: 1576$5962 . \quad$ Retrieved from http://www.redalyc.org/articulo.oa?id=23 1317045002

Grijalva, J. (2009). Liderazgo empresarial. Estado de México. Viveros de Asís 96, Col. Viveros de la Loma, Tlalnepantla, C.P. 54080. Retrieved from www.usfq.edu.ec/publicaciones/polemik a/.../polemika003_017_articulo012.pdf

Hernández, R., Fernández, C., \& Baptista, P. (2014). Metodología de la investigación. Journal of Chemical Information and Modeling (Vol. 53). https://doi.org/10.1017/CBO9781107415 324.004

Mehech, C., Cordero, A., \& Gómez, T. (2016). Medición Del Compromiso Laboral Y Su Impacto En Los Resultados De La Empresa, 1-91. Retrieved from http://repositorio.uchile.cl/bitstream/hand le/2250/139537/Medición del compromiso laboral y su.pdf?sequence=1

Minaya, M. (2014). El liderazgo transformacional de los directivos y las actitudes de los docentes hacia un compromiso organizacional en la institución educativa $N^{\circ}$ 5084. Tesis, $174 . \quad$ Retrieved from http://cybertesis.unmsm.edu.pe/bitstrea $\mathrm{m} /$ handle/cybertesis/3989/Minaya_cm.p df? sequence=1\&i sAllowed=y 
Noriega, M. (2008). La Importancia Del Liderazgo En Las Organizaciones. Ensayo, 12 https://doi.org/10.1017/CBO9781107415 324.004

Robles, J. \&, \& De la Garza, T. (2009). El compromiso organizacional y la actitud de los profesores de ciencias económico administrativas de las IES públicas frente a las necesidades de capacitación institucionales, 2(2), 69-78.

Salvador, E. Sánchez, J. (2018). Liderazgo de los directivos y compromiso organizacional Docente,20(1), 9-18. https://doi.org/10.18271/ria.2018.335
Sun, J., \& Liethwood, K. (2012). Efectos del liderazgo escolar transformacional en el rendimiento de los estudiantes. Revista Iberoamericana Sobre Calidad, Eficacia Y Cambio En Educación., 12(4e), 41-70. Retrieved from file://C:/Users/tpp/Downloads/DialnetEfectosDelLiderazgoEscolarTransforma cionalEnEIRend-5119037.pdf

Tone, M. L. (2017). Liderazgo transformacional y su relacion con el compromiso organizacional en Mibanco, la victoria - 2017, 70. Retrieved from http://repositorio.ucv.edu.pe/bitstream/h andle/UCV/3445/Tone_IML.pdf?sequen ce $=1$ \&isAllowe $d=y$ 